\title{
Effects of the ECG Sampling Frequency on the Multiscale Entropy of Heart Rate Variability
}

\author{
Paolo Castiglioni ${ }^{1}$, Andrea Faini ${ }^{2}$ \\ ${ }^{1}$ IRCCS Fondazione Don Carlo Gnocchi, Milan, Italy \\ ${ }^{2}$ Istituto Auxologico Italiano, IRCCS, Department of Cardiovascular, Neural and Metabolic Sciences, \\ S.Luca Hospital, Milan, Italy
}

\begin{abstract}
It is known that the spectral analysis of heart rate variability requires an ECG sampling frequency $F_{S}>100$ $H z$ with parabolic interpolation to refine the $R$ peak if $F_{S}<250 \mathrm{~Hz}$. By contrast, the effects of quantization errors in Multiscale Entropy (MSE) analysis due to low $F_{S}$ have never been evaluated systematically. Our aim is thus to describe the effects of low $F_{S}$ and parabolic interpolation on MSE. We considered 21 ECG recordings of 10' duration sampled at $500 \mathrm{~Hz}$ (reference). We decimated the ECG to simulate $F_{S}$ between 250 and $50 \mathrm{~Hz}$, we extracted the tachograms without and with parabolic interpolation and estimated MSE at scales between 1 beat (=SampEn) and 50 beats. The estimates were expressed as the percentage of the reference and the error was quantified by the interquartile range (IQR) of their distribution.

SampEn showed high sensitivity to $F_{S}$ with IQR $>10 \%$ at $250 \mathrm{~Hz}$ and $>16 \%$ at $167 \mathrm{~Hz}$; however, the parabolic interpolation dramatically decreased the IQR below $2 \%$ up to $F_{S}=71 \mathrm{~Hz}$. The MSE estimates at larger scales were less sensitive to $F_{S}$ with $I Q R \leq 2 \%$ even at $F_{S}=50 \mathrm{~Hz}$. Thus the ECG sampling rate is more critical for SampEn than for MSE at larger scales and interpolation procedures are required when $F_{S}<250 \mathrm{~Hz}$.
\end{abstract}

\section{Introduction}

Any algorithm for the analysis of heart rate variability (HRV) requires that the position of the $\mathrm{R}$ peak is identified from the ECG signal with sufficient precision. The sampling rate of the ECG influences importantly how well the $\mathrm{R}$ peak is identified, relevantly determining the level of noise affecting the tachogram.

The required minimum level of precision for detecting the position of the $\mathrm{R}$ peak depends on the nature of the HRV analysis. As regards the spectral analysis, the Guidelines on HRV defined the optimal range for the
ECG sampling rate to be between 250 and $500 \mathrm{~Hz}$, recommending to refine the time position of the $\mathrm{R}$ peak with a parabolic interpolation in case of sampling rates between 100 and $250 \mathrm{~Hz}$ [1].

More recently new complexity-based estimators of the heart rate dynamics have been proposed. Among them, the Multiscale Entropy (MSE) is arousing interest because of its ability to detect cardiovascular alterations [2]. The clinical value of MSE has been demonstrated in several HRV studies (see a short review in [3]). However, the effects of errors introduced by low sampling rates have never been evaluated systematically.

Therefore, the aim of this work is to assess the effects on the MSE estimates of low sampling rates of the ECG with and without reconstruction of the $\mathrm{R}$ peak by parabolic interpolation.

\section{Methods}

We considered the ECG recordings in 21 participants (17 women, 4 men) that constitute the EuroBavar data set available at www.eurobavar.altervista.org. Subjects were recorded for about 10-12 minutes twice, in supine and standing position. The ECG was sampled at $500 \mathrm{~Hz}$ with 16 bits resolution (see details on data collection in [4]). To simulate a sampling frequency $\left(F_{s}\right)$ lower than $500 \mathrm{~Hz}$, each ECG was decimated taking 1 sample every 2 samples $\left(F_{s}=250 \mathrm{~Hz}\right), 3$ samples $\left(F_{s}=167 \mathrm{~Hz}\right), 4$ samples $\left(F_{s}=125 \mathrm{~Hz}\right), 5$ samples $\left(F_{s}=100 \mathrm{~Hz}\right), 7$ samples $\left(F_{s}=71\right.$ $\mathrm{Hz})$ and 10 samples $\left(F_{s}=50 \mathrm{~Hz}\right)$.

The $\mathrm{R}$ wave was identified with a derivative-andthreshold algorithm. The tachogram was extracted in two ways: without and with the refining of the position of the $\mathrm{R}$ peak by a parabolic interpolation, which identifies the $\mathrm{R}$ peak as the maximum of the parabola passing through the highest sample of the $\mathrm{R}$ wave and the samples immediately preceding and following the highest sample.

The tachograms extracted from the ECGs at $500 \mathrm{~Hz}$ without parabolic interpolation represent the reference for 


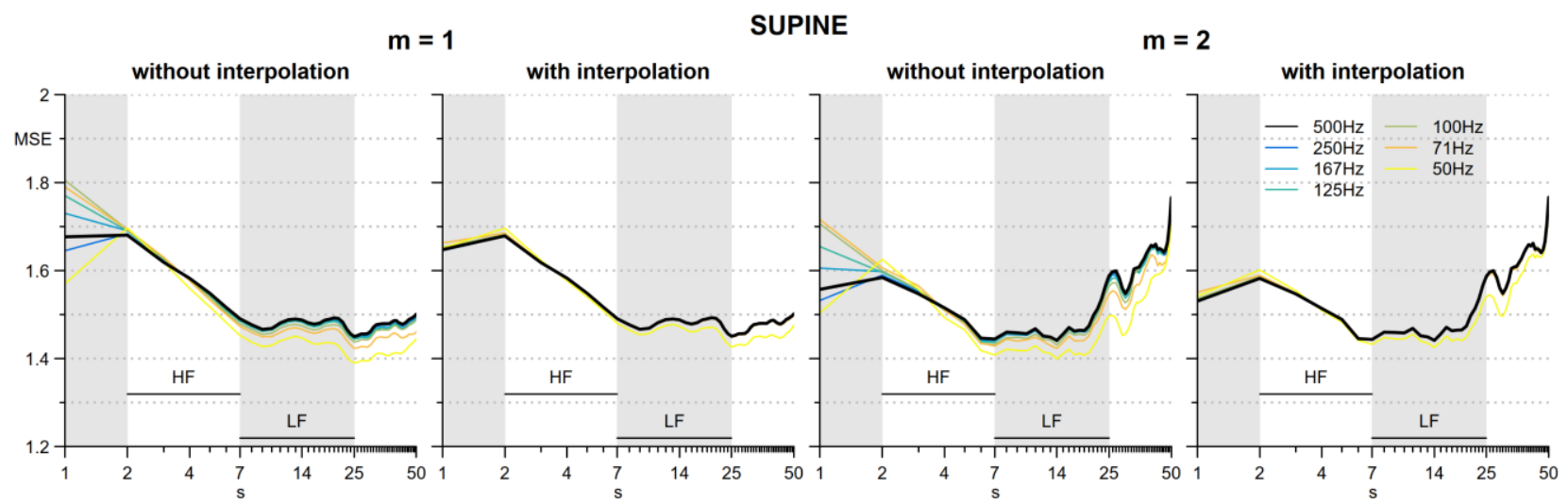

Figure 1. Multiscale entropy MSE in supine posture: mean value over 21 participants. From left to right: embedding dimension $m=1$ without and with refining of the $\mathrm{R}$ peak by the parabolic interpolation; embedding dimension $m=2$ without and with parabolic interpolation. The black line is the reference, estimated from the 500-Hz ECG, colour lines are estimates from progressively decimated ECGs, from 1 every 2 samples $(250 \mathrm{~Hz})$ up to 1 every 10 samples $(50 \mathrm{~Hz})$. The horizontal lines indicate the scale ranges for defining $\mathrm{MSE}_{\mathrm{HF}}$ and $\mathrm{MSE}_{\mathrm{LF}}$.

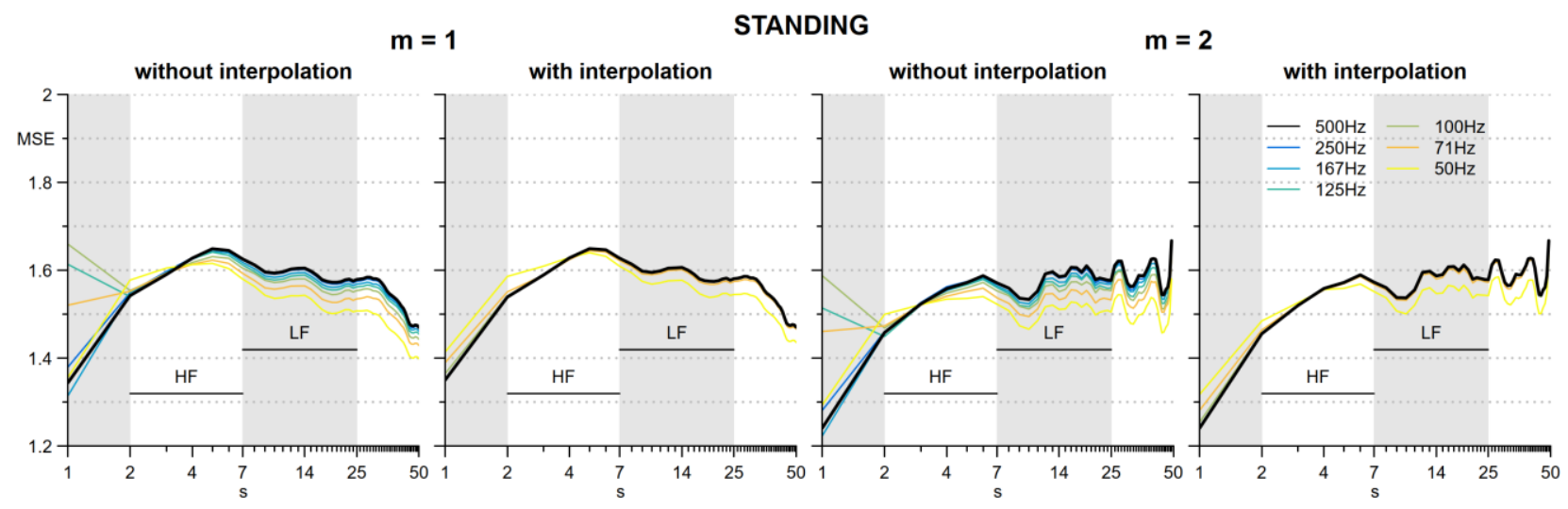

Figure 2. Multiscale entropy MSE in standing posture; see figure 1 for symbols.

studying the effects of the sampling rate without R-peak reconstruction, those extracted with parabolic interpolation are the reference for studying the effects with R-peak reconstruction. Therefore, for each participant and posture (supine or standing), we derived 2 reference tachograms (without and with parabolic interpolation) and 12 decimated tachograms (6 without and 6 with interpolation).

The MSE analysis was performed by the algorithm described in [3] employing a Butterworth filter for coarse graining, with embedding dimensions $m=1$ and $m=2$, setting the tolerance $r$ to $20 \%$ the standard deviation of the time series and applying a fixed-tolerance strategy [5]. The algorithm provided MSE coefficients for scales $s$ between 1 beat (corresponding to the Sample Entropy, SampEn) and 50 beats. To concisely describe the $\operatorname{MSE}(s)$ profile, we defined a short-term MSE index, $\mathrm{MSE}_{\mathrm{HF}}$, average of $\operatorname{MSE}(s)$ over $2 \leq s \leq 7$ beats (approximately corresponding to the high-frequency components of the traditional spectral analysis) and a long-term MSE index,
$\mathrm{MSE}_{\mathrm{LF}}$, average of $\operatorname{MSE}(s)$ over $7<s \leq 25$ beats (approximately corresponding to the low-frequency spectral components).

The estimates of SampEn, $\mathrm{MSE}_{\mathrm{HF}}$, and $\mathrm{MSE}_{\mathrm{LF}}$ were expressed as the percentage of their reference value (thus estimates greater or lower than $100 \%$ indicate an over- or under-estimation error respectively) and reported statistically by the median and the interquartile range over the group. This was done separately for the tachograms with and without interpolation, for each subsampling, each embedding dimension, and each posture.

\section{Results}

Figure 1 shows the profiles of $\operatorname{MSE}(s)$ in the supine position at decreasing sampling rates. SampEn $(s=1)$ is substantially overestimated reaching the largest values for $F_{s}=100 \mathrm{~Hz}$. Deviations from the $500-\mathrm{Hz}$ reference are much lower at $s>2$ and consist in a progressive underestimation as $F_{s}$ decreases. The parabolic 
Table 1. Median [Q3-Q1] of MSE indices in supine as percentage of their reference value at decreasing $F_{s}$.

\begin{tabular}{|c|c|c|c|c|}
\hline \multirow[t]{2}{*}{ Embedding } & \multirow{2}{*}{$\begin{array}{c}\text { Dimension: } \\
\text { without } \\
\text { interpolation } \\
\end{array}$} & \multirow{2}{*}{$\begin{array}{cc}m=1 & \\
& \text { with } \\
\text { interpolation }\end{array}$} & \multicolumn{2}{|c|}{$m=2$} \\
\hline & & & $\begin{array}{l}\text { without } \\
\text { interpolation }\end{array}$ & $\begin{array}{c}\text { with } \\
\text { interpolation }\end{array}$ \\
\hline \multicolumn{5}{|c|}{ SampEn (\% of the reference) } \\
\hline$F_{s}=250 \mathrm{~Hz}$ & 95.3 [91.6-106.2] & 99.9 [99.7-100] & 94.6 [92.5-104.7] & 99.8 [99.6-100.3] \\
\hline$F_{s}=167 \mathrm{~Hz}$ & $100.6[92.2-125.3]$ & 100.1 [99.8-100.2] & 101.3 [92.2-126.4] & 99.8 [99.2-100.2] \\
\hline$F_{s}=125 \mathrm{~Hz}$ & $106.6[94.9-119.7]$ & $100[99.9-100.2]$ & 109.8 [94.4-124.3] & $100.2[99.8-100.7]$ \\
\hline$F_{s}=100 \mathrm{~Hz}$ & $107[93.3-118.5]$ & $100.1[99.9-100.3]$ & $113.1[95.7-118.4]$ & $100.3[99.7-100.8]$ \\
\hline$F_{s}=71 \mathrm{~Hz}$ & 104.2 [90.6-128.4] & 100.2 [100-100.8] & 107.4 [97-131.3] & 101.1 [99.9-102.1] \\
\hline$F_{s}=50 \mathrm{~Hz}$ & $98.7[81.3-107.7]$ & $101[96.8-102.7]$ & 96.9 [85.8-108.9] & $101.2[97.4-103.1]$ \\
\hline \multicolumn{5}{|c|}{$\operatorname{MSE}_{\mathrm{HF}}(\%$ of the reference) } \\
\hline$F_{s}=250 \mathrm{~Hz}$ & $100[99.8-100.1]$ & 100 [100-100] & $100.2[99.8-100.3]$ & $100[99.9-100.1]$ \\
\hline$F_{s}=167 \mathrm{~Hz}$ & $99.9[99.8-100.1]$ & $100[100-100]$ & $100.2[99.8-100.4]$ & $100[99.9-100.1]$ \\
\hline$F_{s}=125 \mathrm{~Hz}$ & 99.9 [99.7-100.1] & $100[100-100]$ & 99.8 [99.6-100.2] & $100[99.9-100.1]$ \\
\hline$F_{s}=100 \mathrm{~Hz}$ & 99.8 [99.4-99.9] & $100[99.9-100]$ & $99.7[99.5-99.9]$ & 100.1 [99.9-100.1] \\
\hline$F_{s}=71 \mathrm{~Hz}$ & 99.7 [99.4-99.9] & $100[99.9-100.1]$ & 99.8 [99.5-100.6] & $100[99.9-100.2]$ \\
\hline$F_{s}=50 \mathrm{~Hz}$ & $98.9[98.7-99.5]$ & $99.8[99.7-100]$ & 99.6 [99-100.4] & 99.9 [99.7-100.7] \\
\hline \multicolumn{5}{|c|}{$\operatorname{MSE}_{L F}(\%$ of the reference) } \\
\hline$F_{s}=250 \mathrm{~Hz}$ & $100[99.9-100.1]$ & 100 [100-100] & $99.9[99.9-100]$ & $100[100-100]$ \\
\hline$F_{s}=167 \mathrm{~Hz}$ & 99.9 [99.6-100] & $100[100-100]$ & 99.7 [99.6-100] & $100[100-100]$ \\
\hline$F_{s}=125 \mathrm{~Hz}$ & 99.8 [99.5-99.9] & $100[100-100]$ & 99.9 [99.4-100] & 100 [100-100] \\
\hline$F_{s}=100 \mathrm{~Hz}$ & $99.7[99.2-99.8]$ & $100[99.9-100]$ & 99.7 [99.2-99.9] & $100[99.9-100]$ \\
\hline$F_{s}=71 \mathrm{~Hz}$ & $99.1[98.4-99.8]$ & $99.9[99.9-100]$ & $99.4[98.5-99.8]$ & $100[99.8-100]$ \\
\hline$F_{s}=50 \mathrm{~Hz}$ & $98.6[97.6-99.3]$ & 99.8 [99.5-99.8] & $98.3[96.9-99.1]$ & $99.7[99.4-99.9]$ \\
\hline
\end{tabular}

interpolation reduces the deviations from the reference and the errors become negligible at all the scales when $F_{s} \geq 100 \mathrm{~Hz}$. In standing (figure 2) MSE is lower than in supine at $s=1$ and greater than in supine at $s>1$, in agreement with a previous study [6]. However, also in standing large discrepancies appear at $s=1$ and the interpolation corrects these errors.

Tables 1 and 2 report the MSE indices as the percentage of their $500-\mathrm{Hz}$ reference. The medians highlight evident overestimation of SampEn at $71 \leq F_{s} \leq 125 \mathrm{~Hz}$ : as to $m=2$ (the more used embedding dimension in HRV) the last quartile of the distribution shows overestimations greater than $24-31 \%$ in supine and $28-47 \%$ in standing. By contrast, evidence of small underestimations appears in the $\mathrm{MSE}_{\mathrm{HF}}$ and $\mathrm{MSE}_{\mathrm{LF}}$ indices at the lowest sampling rates. The interpolation almost corrects the estimation bias and reduces dramatically the amplitude of the interquartile ranges.

\section{Conclusions}

This work reports for the first time that the effects of relatively low ECG sampling rates can be important for MSE estimated at the scale of 1 beat, which corresponds to the popular SampEn estimator of entropy. Unexpectedly, the bias error does not show a monotonic trend with $F_{s}$ : it increases when $F_{s}$ decreases from $500 \mathrm{~Hz}$ up to $100 \mathrm{~Hz}$ but at sampling rates lower than $100 \mathrm{~Hz}$ it starts decreasing. This trend can be explained considering the specific nature of the errors associated with a low sampling rate. On one hand, a low $F_{s}$ introduces a white noise distributed uniformly over the $\Delta \mathrm{T}=1 / F_{s}$ segment centered around the true position of the R-peak: this white noise artifactually increases the estimate of entropy. On the other hand, a low $F_{s}$ also introduces a quantization of the measured R-R intervals, which are a multiple of $\Delta \mathrm{T}$. This reduces the number of possible levels of the tachogram increasing the probability that the series repeats specific values of the R-R intervals eventually decreasing the entropy estimate.

Interestingly, at $s>2$ beats we observed an underestimation of the MSE, being the overestimation (that we hypothesised to be due to the "white noise" error uniformly distributed over $\Delta \mathrm{T}$ ) absent. An explanation for the lack of overestimation at the shorter scales may reside on the fact that the fixed-threshold approach concentrates the unpredictability of stochastic processes without memory, like the white noise, at the shortest scales explaining why a white noise error affects the shortest scales only [5]. By contrast, the varying-threshold approach entrains the irregularity of white noise to the largest scales by adjusting the tolerance. Thus, it is possible that the results would have been different by estimating MSE using the varying-threshold approach, with overestimations affecting the larger scales too.

The parabolic interpolation partially reconstructs the 
Table 2. Median [Q3-Q1] of MSE indices in standing as percentage of their reference value at decreasing $F_{s}$.

\begin{tabular}{|c|c|c|c|c|}
\hline \multirow[t]{2}{*}{ Embedding } & \multirow{2}{*}{$\begin{array}{l}\text { Dimension: } \\
\text { without } \\
\text { interpolation }\end{array}$} & $m=1$ & \multicolumn{2}{|c|}{$m=2$} \\
\hline & & $\begin{array}{c}\text { with } \\
\text { interpolation }\end{array}$ & $\begin{array}{c}\text { without } \\
\text { interpolation }\end{array}$ & $\begin{array}{c}\text { with } \\
\text { interpolation }\end{array}$ \\
\hline \multicolumn{5}{|c|}{ SampEn (\% of the reference) } \\
\hline$F_{s}=250 \mathrm{~Hz}$ & $109.2[92.9-111.1]$ & $100[99.9-100.1]$ & $108.1[95.4-112.2]$ & $100.1[99.8-100.4]$ \\
\hline$F_{s}=167 \mathrm{~Hz}$ & $89.1[85.3-101.3]$ & $99.9[99.9-100.1]$ & 89 [86.8-105.7] & $99.8[99.7-100.4]$ \\
\hline$F_{s}=125 \mathrm{~Hz}$ & $133.5[97.8-142.7]$ & $100[99.8-100.2]$ & 138 [97.3-147] & $100[99.5-101.1]$ \\
\hline$F_{s}=100 \mathrm{~Hz}$ & $126.5[117.2-137.4]$ & 100.2 [100.1-100.7] & $131.6[117.5-143.8]$ & 100.7 [100.4-101.2] \\
\hline$F_{s}=71 \mathrm{~Hz}$ & 111.9 [104.7-124.4] & 100.8 [100.1-101.6] & $116.8[109-128.5]$ & $102.2[101.5-102.9]$ \\
\hline$F_{s}=50 \mathrm{~Hz}$ & $97.1[90.3-110.4]$ & 100.5 [98.7-104.3] & $103[94.3-113.6]$ & 105.9 [101-109.6] \\
\hline \multicolumn{5}{|c|}{$\operatorname{MSE}_{\mathrm{HF}}(\%$ of the reference $)$} \\
\hline$F_{s}=250 \mathrm{~Hz}$ & $100[99.9-100.1]$ & $100[100-100]$ & $100[99.9-100.3]$ & $100[99.9-100.1]$ \\
\hline$F_{s}=167 \mathrm{~Hz}$ & $100[99.9-100.1]$ & $100[100-100]$ & $99.9[99.7-100.1]$ & $100[99.9-100.1]$ \\
\hline$F_{s}=125 \mathrm{~Hz}$ & $99.9[99.8-100]$ & $100[100-100.1]$ & $99.9[99.7-100.2]$ & $100[100-100.1]$ \\
\hline$F_{s}=100 \mathrm{~Hz}$ & $99.9[99.8-100.1]$ & $100[99.9-100]$ & $99.9[99.7-100.3]$ & $100[99.9-100.1]$ \\
\hline$F_{s}=71 \mathrm{~Hz}$ & $99.6[99.2-99.8]$ & $100[99.9-100]$ & $99.8[99.3-100.1]$ & $100.1[99.8-100.1]$ \\
\hline$F_{s}=50 \mathrm{~Hz}$ & $98.9[98.6-99.4]$ & 99.7 [99.6-99.9] & 99 [98.3-99.9] & $99.6[99.4-100]$ \\
\hline \multicolumn{5}{|c|}{$\mathrm{MSE}_{\mathrm{LF}}(\%$ of the reference) } \\
\hline$F_{s}=250 \mathrm{~Hz}$ & 100 [99.9-100] & $100[100-100]$ & $100[99.9-100]$ & $100[100-100]$ \\
\hline$F_{s}=167 \mathrm{~Hz}$ & 99.9 [99.8-99.9] & $100[100-100]$ & $99.9[99.7-100]$ & $100[100-100]$ \\
\hline$F_{s}=125 \mathrm{~Hz}$ & 99.8 [99.7-99.9] & $100[100-100]$ & $99.8[99.6-100]$ & $100[100-100]$ \\
\hline$F_{s}=100 \mathrm{~Hz}$ & 99.6 [99.6-99.8] & $100[100-100]$ & $99.7[99.5-99.9]$ & 100 [99.9-100] \\
\hline$F_{s}=71 \mathrm{~Hz}$ & $99.3[99-99.4]$ & 99.9 [99.8-100] & $99.3[98.8-99.7]$ & $99.9[99.8-100]$ \\
\hline$F_{s}=50 \mathrm{~Hz}$ & $98.6[97.9-99.2]$ & 99.7 [99.4-99.9] & $98.5[97.6-99]$ & $99.6[99.2-99.9]$ \\
\hline
\end{tabular}

R-peak reducing the noise distributed over $\Delta \mathrm{T}$ and it also allows the tachogram to have any real value and not multiples of $\Delta \mathrm{T}$ only. The present study shows that this refining procedure works remarkably well and that the MSE estimates present negligible errors at all the scales even with ECG sampling rates of $100 \mathrm{~Hz}$ only.

\section{References}

[1] Task Force of the European Society of Cardiology and the North American Society of Pacing and Electrophysiology, 'Heart rate variability. Standards of measurement, physiological interpretation, and clinical use.', Eur.Heart J., vol. 17, no. 0195-668X, pp. 354-381, Mar. 1996.

[2] M. Costa, A. L. Goldberger, and C.-K. Peng, 'Multiscale entropy analysis of biological signals', Phys. Rev. E, vol. 71, no. 2, p. 021906, Feb. 2005, doi: 10.1103/PhysRevE.71.021906.

[3] P. Castiglioni, G. Parati, and A. Faini, 'Informationdomain analysis of cardiovascular complexity: night and day modulations of entropy and the effects of hypertension', Entropy, vol. 21, no. 6, p. 550, May 2019, doi: 10.3390/e21060550.

[4] D. Laude et al., 'Comparison of various techniques used to estimate spontaneous baroreflex sensitivity (the EuroBaVar study)', Am. J. Physiol. Regul. Integr. Comp. Physiol., vol. 286, no. 1, pp. R226-231, Jan. 2004, doi: 10.1152/ajpregu.00709.2002.

[5] P. Castiglioni, P. Coruzzi, M. Bini, G. Parati, and A. Faini, 'Multiscale sample entropy of cardiovascular signals: does the choice between fixed- or varying-tolerance among scales influence its evaluation and interpretation?', Entropy, vol. 19, no. 11, p. 590, Nov. 2017, doi: 10.3390/e19110590.

[6] Z. Turianikova, K. Javorka, M. Baumert, A. Calkovska, and M. Javorka, 'The effect of orthostatic stress on multiscale entropy of heart rate and blood pressure', Physiol. Meas., vol. 32, no. 9, pp. 1425-1437, Jul. 2011, doi: 10.1088/0967-3334/32/9/006.

Address for correspondence:

Paolo Castiglioni.

IRCCS Fondazione Don C. Gnocchi

Via Capecelatro 66, I 20148, Milano, Italy.

pcastiglioni@dongnocchi.it 